\title{
Erratum
}

\section{Erratum to: Modeling Parkinson's Disease in C. elegans}

Jason F. Cooper ${ }^{\mathrm{a}}$ and Jeremy M. Van Raamsdonk $\mathrm{k}^{\mathrm{a}, \mathrm{b}, \mathrm{c}, *}$

${ }^{a}$ Laboratory of Aging and Neurodegenerative Disease, Center for Neurodegenerative Science, Van Andel Research Institute, Grand Rapids, MI, USA

${ }^{\mathrm{b}}$ Department of Neurology and Neurosurgery, McGill University, Montreal, QC, Canada

${ }^{\mathrm{c}}$ Metabolic Disorders and Complications Program, and Brain Repair and Integrative Neuroscience Program, Research Institute of the McGill University Health Centre, Montreal, QC, Canada

[Journal of Parkinson's Disease 8(1), 2018, 17-32, DOI 10.3233/JPD-171258]

https://content.iospress.com/articles/journal-of-parkinsons-disease/jpd171258

In the original publication, the images for Figure 2 and Figure 3 were reversed. 